\section{Sharing Responsibility - A Holistic Approach to Addressing Early Childhood Caries}

Oral health is an integral component of overall general health. Poor oral health can profoundly affect a child's health and general well-being. Dental caries is the most common chronic childhood disease, being five times more common than asthma. It is an infectious, transmissible disease with significant detrimental effects, if left untreated.

Early childhood caries (ECC) formerly termed as "nursing bottle caries" is a particularly virulent form of caries that affects primary dentition. It begins soon after eruption and progresses rapidly. Untreated caries can affect a child's growth, result in significant pain and potentially life threatening infection, and diminish overall quality of life $[1,2]$.

Risk factors which predispose to ECC include (i) early colonization of mutans streptococci; (ii) lack of oral hygiene routines; (iii) children who sleep with bottle or breastfeed throughout the night; (iv) high frequency of sugar consumption; $(v)$ mothers with high caries rate; (vi) low socioeconomic status; and (vii) low parental education [3].

Timely identification of risk factors and appropriate intervention; however, can prevent this disease. Early intervention provides the opportunity to examine the child before the initiation of dental caries and educate parents about appropriate oral hygiene and feeding practices. It is recommended that the first dental visit should occur at or around one year of age [4].

In India; however, very few children visit a dentist before 4 or 5 years of age and the first visit is usually a 'problem' visit with the child reporting most often with pain or swelling. Due to the lack of access to parents of infants and toddlers, pediatric dentists are unable to provide timely preventive guidance.

Pediatricians on the other hand see a large number of children during their infant and toddler years and can play a significant role in prevention and control of ECC. Establishing collaborative relationship with these health professionals is therefore, essential for increasing access to dental care [5].

An initial step in this regard can be integrating preventive oral health care with the immunization visits of the child. During these visits, pediatricians can provide counseling on caries prevention and encourage parents to make well-child visit to a dentist. The inclusion of oral health status of the child in the immunization-card can serve as record of the preventive oral health services provided and a reminder for subsequent interventions.

Namrata C Gill
Department of Pedodontics and Preventive Dentistry,
Dr. HSJ Institute of Dental Sciences and Hospital,
Panjab University, Chandigarh, India.
namratagill@gmail.com

\section{REFERENCES}

1. American Academy on Pediatric Dentistry. Policy on Early Childhood Caries (ECC): Classifications, Consequences, and Preventive Strategies. Available from: URL: http:// www.aapd.org/media/policies_guidelines/ p_eccclassifications.pdf. Accessed June 14, 2012.

2. American Academy on Pediatric Dentistry. Guideline on Infant Oral Health Care. Available from: URL: http:// www.aapd.org/media/policies_guidelines/ g_infantoralhealthcare.pdf. Accessed June 14, 2012.

3. Hale KJ. American Academy of Pediatrics Section on Pediatric Dentistry. Oral Health Risk Assessment Timing and Establishment of the Dental Home. Pediatrics. 2003;111:1113-6.

4. Lee JY, Bouwens TJ, Savage MF, Vann WF Jr. Examining the cost-effectiveness of early dental visits. Pediatr Dent. 2006;28:102-5.

5. Section on Pediatric Dentistry and Oral Health. Preventive Oral Health Intervention for Pediatricians. Pediatrics. 2008;122:1387-94. 